\title{
Use of lasers in assisted fertilization and hatching
}

\author{
A.Obruca ${ }^{1}$, H.Strohmer ${ }^{1}$, D.Sakkas ${ }^{2}$, Y.Menezo ${ }^{2,3}$, \\ A.Kogosowski $^{4}$, Y.Barak ${ }^{4}$ and W.Feichtinger ${ }^{1,5}$ \\ 'Institute of Sterility Treatment, Trauttmansdorffgasse 3A, \\ A-1130 Vienna, Austria, ${ }^{2}$ Hôpital Cantonal Universitaire de \\ Genève, Switzerland, ${ }^{3}$ Institut Rhônalpin, Lyon, France and \\ ${ }^{4}$ Herzliya Medical Centre, Herzliya-on-Sea, Israel \\ ${ }^{5}$ To whom correspondence should be addressed
}

The erbium-yttrium - aluminium-garnet (Er:YAG) laser has been applied to micromanipulation in humans. It was used in the fertilization process for both subzonal insemination (SUZI) and for partial zona dissection (PZD). Laser-assisted micromanipulation achieved significantly higher fertilization rates $(34.8 \%)$ when compared to mechanical SUZI (16.1\%), but use of the laser did not improve the PZD results (laser $14.8 \%$ versus mechanical $14 \%$ ). The Er:YAG laser was used to assist hatching. In the mouse it significantly improved the hatching rate (80 versus $29.3 \%$ ) $110 \mathrm{~h}$ after administration of human chorionic gonadotrophin. This technique was applied in two different centres to patients with previous invitro fertilization (IVF) failures. The implantation rate per embryo (14.4\% laser-assisted hatching versus $6 \%$ control group) and the pregnancy rate per transfer (40 versus $16.2 \%$ ) were improved.

Key words: assisted hatching/Er:YAG laser/in-vitro fertilization/ laser micromanipulation/zona pellucida

\section{Introduction}

Since Cohen et al. (1988) reported the first pregnancy after mechanical partial zona dissection (PZD), this micromanipulation technique has been used routinely to enhance the chance of fertilizing the ovum in instances of poor sperm quality (Tucker et al., 1991). Recently, micromanipulation was performed to facilitate embryonic hatching (Cohen, 1991; Cohen et al., 1992). The increasing importance of lasers in medicine has encouraged us to consider the application of this technology for zona drilling, subzonal insemination (SUZI) and assisted hatching in our invitro fertilization (IVF) programme.

However, it is recommended that the following four criteria are taken into account when using lasers in human embryology (Welch et al., 1991): (i) absolute avoidance of thermic effects; (ii) prevention of genetic damage by a wavelength sufficiently distant from the absorption maximum of DNA; (iii) low ablation threshold to ensure precision and to minimize mechanical vibration; (iv) easy handling using existing micromanipulation equipment as briefer manipulations are always more efficient.
Special laser equipment fulfilling all these criteria has been developed in our laboratory (Feichtinger et al., 1992; Strohmer and Feichtinger, 1992a)

In this paper, we present the use of the erbium-yttriumaluminium-garnet (Er:YAG) laser in two types of micromanipulation in human IVF technology, i.e. SUZI and PZD. For assisted hatching, a control experiment was tested in the mouse and then applied in humans, particularly in patients with repeated failure of embryo transfer.

\section{Materials and methods}

\section{Laser technology}

The laser system used consisted of an Er:YAG laser (Lisa Laser Products, Katlenburg, Germany) and a laser fibre of $20 \mu \mathrm{m}$ tip diameter. The application of low energy resulted in photoablation-a non-thermic effect of the laser on biological tissue. A wavelength of $2.9 \mu \mathrm{m}$ was selected to avoid damaging the genetic structure of the embryo (Strohmer and Feichtinger, 1992b), yet offering a high absorption in water. At $2.9 \mu \mathrm{m}$ wavelength, penetration depth in biological tissue is about $3 \mu \mathrm{m}$, creating an ablation of the zona pellucida in layers by laser pulses of $\sim 10 \mu \mathrm{J}$ energy. Additionally, the specially developed glass fibre allowed a procedure similar to conventional mechanical PZD.

\section{Micromanipulation}

Two hydraulic fine-control micromanipulators (Narishige, Tokyo, Japan) mounted on a Leitz Labovert inverted microscope (Leitz, Wetzlar, Germany) were used in conjunction with a Hoffmann Modulation Contrast System (Modulation Optics, Greenvale, NY, USA).

All oocytes or embryos to be treated were placed simultaneously on a microscope slide and covered with paraffin oil. During the procedure, the oocyte or embryo was held by negative pressure using a holding pipette of $130 \mu \mathrm{m}$ outer and $20 \mu \mathrm{m}$ inner diameter. The glass fibre, fitted to the manipulator by a pipette holder, was brought in direct contact with the zona, but the tip was not forced further into it. This prevented any contact of the fibre with the surface of the ooplasm or blastomere once it penetrated the zona. Five to eight pulses were necessary to penetrate the zona, creating a $20-30 \mu \mathrm{m}$ opening. The efficiency of the system was easily controlled and visualized by inserting the fibre tip into the hole.

\section{Laser SUZI}

These techniques were performed in cases of male subfertility that had been defined according to World Health Organization 
criteria (WHO, 1987). In most of the cases, there had been at least one preceding IVF attempt, with failure of fertilization. For laser SUZI, a double micro-instrument holder was used with the laser fibre and one thin, but not sharpened, glass pipette. The main advantage of this system was that after a hole had been created by the laser, SUZI was performed easily without mechanical manipulations and uncontrolled sheering forces. Mechanical SUZI was carried out in a number of control cases.

\section{Laser zona drilling}

One hole $(20 \mu \mathrm{m}$ diameter) was made in the zona before conventional in-vitro insemination. The protocol was performed on patients with poor semen parameters, similar to the patients selected for SUZI.

\section{Mouse experiments with laser-assisted hatching}

The embryo culture media used in this study were M16 medium containing $4 \mathrm{mg} / \mathrm{ml}$ of type $V$ bovine serum albumin (BSA; Sigma Pharmaceuticals, Buchs, Switzerland) and M2 HEPES buffered medium (Whittingham, 1971). One-cell embryos were obtained from 3- to 4-week-old C57/bl (IFFA-CREDO, L'Arbresle, France) female mice. Ovulation was induced as previously described (Sakkas and Trounson, 1990). One-cell embryos were obtained $21 \mathrm{~h}$ after administration of human chorionic gonadotrophin (HCG) by tearing the ampulla and releasing the cumulus cell complex plug in M2 medium containing $1 \mathrm{mg} / \mathrm{ml}$ of hyaluronidase type IV (Sigma Pharmaceuticals). The embryos were incubated at $37^{\circ} \mathrm{C}$ in $5 \% \mathrm{CO}_{2}$ in air. Between 66 and 68 $\mathrm{h}$ after $\mathrm{HCG}$ at the 8-cell stage, the assisted hatching procedure was performed according to the following protocol. Groups of 10-15 embryos were placed under paraffin oil in M2 medium on two slides. One slide was left on a warming stage while on the other slide the embryos were subjected to the laser treatment: one hole of the size of the probe was made in the zona pellucida. Once the procedure was finished (10 min per group), the embryos from the two slides were washed in M16 medium and placed in culture droplets in their respective groups. Embryos were subsequently assessed for the number reaching the blastocyst stage and for their ability to expand and hatch at $110 \mathrm{~h}$ after HCG. In a short series, blastocysts were transferred to the uteri of day 3 pseudopregnant recipients. To each uterine horn of each recipient, six embryos from the control and treatment groups were transferred. Recipient females were examined on day 15 to count the number of implantation sites and the developing fetuses.

\section{Laser-assisted hatching}

Assisted hatching was performed in two different IVF centres (Institut für Sterilitätsbetreuung, Vienna, Austria, and Herzliya Medical Centre, Herzliya-on-Sea, Israel) on 129 patients who had had repeated IVF failures. In both centres, the results were compared to a matched control group which consisted of IVF patients with repeated failures of transfer, treated in both centres before the laser was introduced. The mean number of repeated failures of transfer (3.6 assisted hatching versus 3.1 control group) and the mean age were not significantly different between the groups ( 33.8 versus 34.3 years). There were also no differences in ovulation induction and sperm treatment. The number of previous embryos transferred was 11.8 in the laser-treated group
Table I. Micromanipulation in cases of male factor infertility

\begin{tabular}{|c|c|c|c|c|}
\hline & $\begin{array}{l}\text { No. of } \\
\text { treated } \\
\text { oocytes }\end{array}$ & $\begin{array}{l}\text { No. of } \\
\text { fertilized } \\
\text { oocytes }(\%)\end{array}$ & $\begin{array}{l}\text { No. of } \\
\text { embryo } \\
\text { transfers }\end{array}$ & $\begin{array}{l}\text { No. of } \\
\text { pregnancies } \\
\text { (\% per embryo } \\
\text { transfer) }\end{array}$ \\
\hline Mechanical PZD & 57 & $8(14.0)$ & 3 & 0 \\
\hline Laser PZD & 149 & $22(14.8)$ & 14 & $1(7.1)$ \\
\hline Mechanical SUZI & 143 & $23(16.1)$ & 13 & $3(23.1)$ \\
\hline Laser SUZI & 23 & $8(34.8)^{*}$ & 5 & $1(20)^{* * *}$ \\
\hline
\end{tabular}

*Significantly different from other values in the same column. $P<0.05$ **Not significantly different from mechanical SUZI value. PZD = partial zona dissection: SUZI = subzonal insemination.

Table II. Assisted hatching of mouse embryos by Er:YAG laser (AHL), $110 \mathrm{~h}$ post-HCG

\begin{tabular}{llc}
\hline & \multicolumn{2}{l}{ Number (\%) reaching each stage } \\
\cline { 2 - 3 } Stage & Control & AHL \\
\hline Degenerated blastocysts & $20(34.5)$ & $5(9.0)$ \\
Expanded blastocysts & $21(36.2)$ & $6(10.9)$ \\
Hatched (day 4.5 post-HCG) & $17(29.3)$ & $44(80.0)^{*}$ \\
Total & $\mathbf{5 8}$ & $\mathbf{5 5}$ \\
\hline
\end{tabular}

*Significantly different from control value. $P=0.0001$.

$\mathrm{HCG}=$ human chorionic gonadotrophin.

versus 9 in the matched control group. The laser technology assisted in making one hole of the size of the fibre tip, at the 2 - to 4-cell stage $48 \mathrm{~h}$ post-insemination. The embryos were transferred immediately after the laser-assisted hatching.

\section{Statistical analysis}

The results were analysed using the $\chi^{2}$ test and unpaired $t$-test. A standardized computer program (StatView; Abacus Concepts, Berkeley, CA, USA) was applied.

\section{Results}

\section{SUZI and zona drilling}

The results of micromanipulation procedures in male factor infertility patients are summarized in Table I. The best results were obtained with laser SUZI (34.8\% fertilization rate), which was significantly better $(P<0.05)$ than mechanical SUZI ( $16.1 \%)$. However, the pregnancy rate per transfer was similar for both groups (20 versus $23.1 \%$ ). The results of laser-assisted PZD were as poor as those of the mechanical method: fertilization rate 14.8 versus $14 \%$; pregnancy rate 7.1 versus $0 \%$.

\section{Assisted hatching in the mouse}

Laser-assisted hatching shortened the hatching time by comparison with the control group. At day 4.5 post-HCG, $80 \%$ of the mouse embryos had already started the hatching process in the laser-treated group versus $29.3 \%$ in the control group (Table II).

In the short series of embryo transfers in day 3 synchronized pseudopregnant recipients, no differences were observed between the laser-treated embryos and the control group (control group: 
Table III. Results from assisted hatchıng by Er-YAG laser (AHL) in patients with repeated IVF failure in two centres (Institut für Sterilitätsbetreuung, Austria, and Herzliya Medical Centre, Israel)

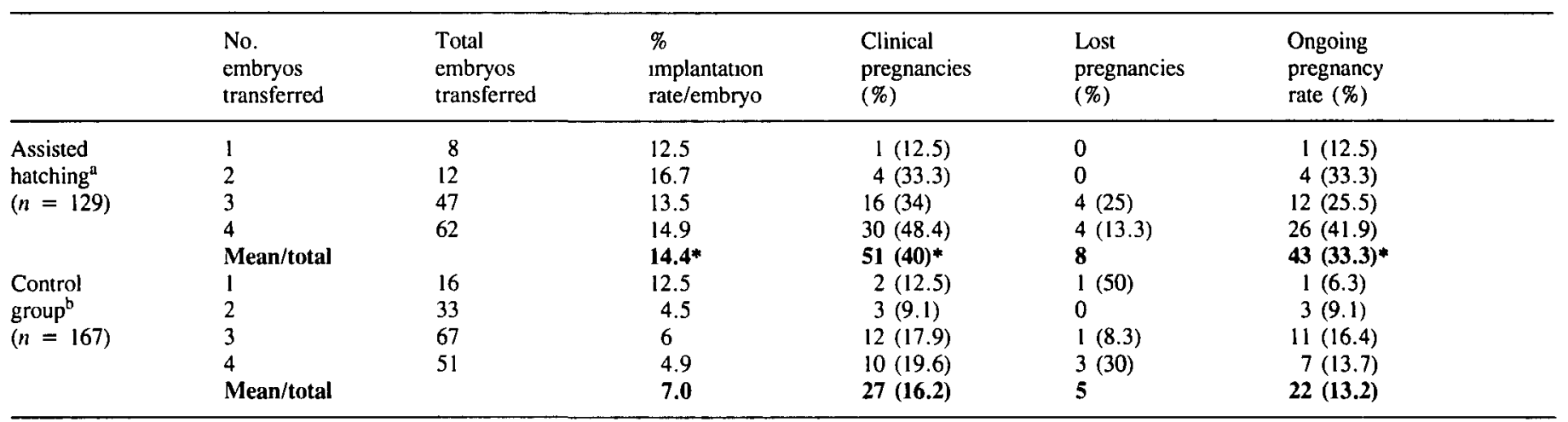

${ }^{a}$ Patients with repeated IVF failures from 1992/1993

bPatients with repeated IVF failures before the laser was introduced.

*Significantly different from control values, $P<0.05$.

two fetuses and four resorptions out of 12 embryos transferred; laser group: three fetuses and four resorptions out of 12 embryos transferred).

\section{Assisted hatching in humans}

Laser-assisted hatching in 129 patients who had had repeated IVF failures was compared with 167 controls who were treated before the laser was introduced. The number of previously failed attempts for the two groups was $3.6 \pm 3$ and $3.1 \pm 2.6$ respectively, and the corresponding number of transferred embryos was $11.8 \pm 12.1$ and $9 \pm 9.2$ respectively. The mean ages of the two groups were similar: $33.8 \pm 5.4$ and $34.3 \pm$ 5. Laser treatment increased the implantation rate per embryo (14.4 versus $7 \%$ ) as well as the clinical pregnancy rate per embryo transfer (40 versus $16.2 \%$ ) and the ongoing pregnancy rate per embryo transfer (33.3 versus $13.2 \%$ ) (Table III). The increase was not related to differences of age, stimulation, sperm treatment or number of embryos transferred.

So far (November 1993), 44 children have been born from 37 patients who had laser-assisted micromanipulation.

\section{Discussion}

Application of ultraviolet (UV) lasers in non-contact mode has been proposed by Tadir et al. (1993) for different micromanipulation procedures. These systems have the advantage that there is no need for disposable fibres and for sterilization; also, there is the possibility of intracellular application. However, certain wavelengths in the UV range bear potential hazards for the genetic structure, the question of heat deposition by these systems has yet to be determined, and the spot size is limited by the wavelength and optics. The creation of large gaps in the zona requires longer exposure and higher laser energies. Last but not least, expensive optics are needed for the microscope. Basic studies in laser-gamete interaction have to be carried out in order to determine the potential hazards of UV lasers. Therefore, these systems are not ready to be applied to humans yet (Neev et al., 1993; Li et al., 1993).

We intended to avoid such problems by using a totally different system: an infrared laser in contact mode. Although disposable or sterilizable fibres are needed, this system, which uses existing micromanipulation equipment, is simple, and the micromanipulation process can be quickly carried out. The spot size can be modulated by the fibre size $(3-30 \mu \mathrm{m})$; very low energies and few pulses are needed to achieve appropriate zona piercing.

However, for assisted fertilization (SUZI and zona drilling) the results are as disappointing as for mechanical processes. This confirms that these technologies are not really improving the fertilization process for the treatment of male subfertility (Sakkas et al., 1992; Ord et al., 1993).

Er:YAG laser-assisted hatching shortens the hatching time in the mouse; in this case, our data are comparable to those described by Neev et al. (1993) for acidic cutting of the zona, but not for laser drilling. UV and Er:YAG laser give totally different results in this case, as confirmed by the degeneration rate observed by the authors. Er:YAG laser treatment does not prevent further implantation when compared to controls.

Used in human IVF, laser-assisted hatching improves both the implantation rate per embryo and the pregnancy rate per transfer. The increases were effective for the two different centres involved in the protocol. So far, 37 patients have delivered 44 healthy children using these laser experiments, and these are the first cases reported in the literature.

The Er:YAG laser offers an alternative method of treatment for repeated failures of embryo transfer. It can be considered as safe and efficient as the assisted hatching using acidic Tyrode proposed by Cohen (1991). However, further evaluations in a totally randomized protocol are necessary to confirm the efficiency of this technology and to define for which indications it is especially useful and can be recommended, as already proposed by Cohen et al. (1992).

\section{References}

Cohen,J. (1991) Assisted hatching of human embryos. J. In Vitro Fertil. Embryo Transfer, 8, 179-190.

Cohen,J., Malter,H., Fehilly,C., Wright,G., Elsner,C., Kort,H. and Massey,J. (1988) Implantation of embryos after partial opening of oocyte zona pellucida to facilitate sperm penetration. Lancet, ii, 162.

Cohen,J., Alikani,M., Trowbridge,J. and Rosenwaks,Z. (1992) 


\section{A.Obruca $e t$ al.}

Implantation enhancement by selective assisted hatching using zona drilling of human embryos with poor prognosis. Hum. Reprod., 7, 685-691.

Feichtinger,W., Strohmer,H., Fuhrberg,P., Radivojevic,K., Antinori,S., Pepe,G. and Versaci,C. (1992) Photoablation of oocyte zona pellucida by erbium-yag laser for in-vitro fertilisation in severe male infertility. Lancet, 339, 811 .

Li,L., Munne,S., Licciardi,F., Neev,J., Tadir,Y., Berns,M., Godke,R. and Cohen,J. (1993) Microinjection of FITC-dextran into mouse blastomeres to assess topical effects of zona photoablation. Zygote, $1,43-48$.

Neev,J., Gonzalez,A., Licciardi,F., Alikani,M., Tadir,Y., Berns,M. and Cohen,J. (1993) Opening of the mouse zona pellucida by laser without a micromanipulator. Hum. Reprod., 8, 939-944.

Ord,T., Patrizio,P., Balmaceda,J.P. and Asch,R.H. (1993) Can severe male factor infertility be treated without micromanipulation? Fertil. Steril., 60, $110-115$.

Sakkas,D. and Trounson,A.O. (1990) Co-culture of mouse embryos with oviduct and uterine cells prepared from mice at different days of pseudopregnancy. J. Reprod. Fertil., 90, 109-118.

Sakkas,D., Lacham,O., Gianaroli,L. and Trounson,A. (1992) Subzonal sperm microinjection in cases of severe male factor infertility and repeated in vitro fertilization failure. Fertil. Steril., 57, $1279-1288$.

Strohmer,H. and Feichtinger,W. (1992a) Successful clinical application of laser for micromanipulation in an in vitro fertilization program. Fertil. Steril., 58, 212-214.

Strohmer,H.and Feichtinger,W. (1992b) Application of laser for micromanipulation: relevance of biophysical criteria. Fertil. Steril., Abstr. Suppl. 48, S40.

Tadir,Y., Neev,J., Ho,P. and Berns,M.W. (1993) Laser for gamete micromanipulation: basic concepts. J. Assist. Reprod. Genet., 10, $121-125$.

Tucker,M.J., Bishop,F.M., Cohen,J., Wiker,S.R. and Wright,G. (1991) Routine application of partial zona dissection for male factor infertility. Hum. Reprod., 6, 676-681.

Welch,A.J., Motamedi,M., Rastegar,S., Le Carpentier,G.L. and Jansen,D. (1991) Laser thermal ablation. Photochem. Photobiol. , 53, 815-823.

Whittingham,D.G. (1971) Culture of mouse ova. J. Reprod. Fertil., 14 (Suppl.), 7-21.

World Health Organization (1987) WHO Laboratory Manual for the Examination of Human Semen and Semen-Cervical Mucus Interaction. 2nd edn. The Press Syndicate of the University of Cambridge, Cambridge.

Received on December 7, 1993; accepted on May 10, 1994 